HStud 23 (2009)1, 37-45

DOI: 10.1556/HStud.23.2009.1.4

\title{
DAS WASSER ALS TECHNISCHES MATERIAL UND ELEMENT IN DER UNGARISCHEN GARTENKUNST UND REPRÄSENTATION DES 18. JAHRHUNDERTS
}

\author{
KATALIN CZIBULA \\ Eötvös-Loránd-Universität, Budapest \\ Ungarn
}

\begin{abstract}
Der Beitrag beschäftigt sich mit der Geschichte der Wasserkultur und deren architektonischen Implementierung im Zeitalter des Barock. In dieser Epoche scheint mir vor allem interessant, was der Mensch über das Wasser als physikalisches Element und dessen Gesetze im Königreich Ungarn überhaupt wusste. Eine der bedeutenden Quellen ist dabei das handschriftliche, enzyklopädisch angelegte Lehrbuch eines ungarischen Piaristen, der darin die zeitgenössischen Erkenntnisse der Naturwissenschaften zusammenzufassen versucht hatte. Es handelt sich dabei um Lukács Mösch's Skriptum Bibliothecae mathematicae ... Classis I-III. Anno 1684. Diese Handschrift unterlegt die wissenschaftlichen Erklärungen auch mit zahlreichen wertvollen Illustrationen, von denen einige hier wiedergegeben werden. Die praktischen Anwendung dieses Mösch'schen Lehrstoffes sehen wir in der ungarischen Gartenkunst des Zeitalters, in der die technische Attraktionen mit dem Theater und der christlichen Symbolik verbunden wurden. Die Beispiele dafür bilden die aristokratischen Gärten von Eger, Cseklész, Pozsonyivánka und Eszterháza, die in diesem Text näher besprochen werden.
\end{abstract}

Schlüsselwörter: Aufklärung, Wasserkunst, Naturwissenschaften, Theater, Gartenkunst, Mythologie

\section{Das Wasser als Ursymbol}

Die Interpretation des Wassers außerhalb der Sphäre des Praktischen, als Urmaterie, als Analogie, als Symbol ist so alt wie das menschliche Denken selbst. „Jedes Element eignet sich dazu, metaphysisch behandelt zu werden und das vollkommene Verständnis für die Welt vermittels Analogien zu antizipieren - aber es gibt kein Element, das geeigneter wäre als das Wasser, schon allein, weil Leben wasserartiger ist als luft- oder feuerartig. Das Leben ist produktiv und mütterlich und sensibel, und es gibt kein Lebewesen, dessen Organismus nicht zum Großteil aus Wasser bestünde."1 
In Europa wird Thales als Ausgangspunkt des philosophischen Denkens angenommen, der das Wasser als Urmaterie bezeichnet hatte und den Ursprung der Welt auf das Wasser zurückführte.

\begin{abstract}
Thales ging davon aus, dass die Welt voller Dämonen sei - daimones - und von einem Gott, einem Weltgeist gelenkt werde. Diese göttliche Kraft gibt dem Urgewässer Leben. Dies war ein großer Schritt nach vorn: die kosmologische Erklärung basierte nun nicht mehr auf einer Geschichte, basiert nicht mehr auf den mehr oder weniger amorphen oder mit den großen Grundprinzipien identischen absichtlichen Handlungen der Götter. Den Eigenschaften der Urmaterie gemäß ist sie in diversen Formen vorhanden. Diese Theorie verkündete die Einheit des Lebens, egal, ob zudem noch eine „beseelte Welt" darin einen Platz bekommt, oder nicht. Sie öffnet einem beispiellosen Gedanken Tür und Tor. ${ }^{2}$
\end{abstract}

Nachdem also Wasser bereits in der ägyptischen, babylonischen, sumerischen Mythologie gleichermaßen eine Schlüsselrolle spielte, erscheint sie auch in der griechisch-römischen Mytghologie als eine Urkraft, und hat sowohl im Alten als auch im Neuen Testament hervorgehobenen Symbolstatus. Die Vorläufer dieser Symbolik sind in der griechisch-römischen Mythologie beheimatet, in Bezug auf das Christentum ist aber das jüdische Vorbild, wonach Wasser zur Reinigung dient, das ausschlaggebende. So wird das Wasser und die damit in Verbindung stehende Quelle, der Brunnen, zum Begriff der Reinheit, der Genesung, später das Wasser des Lebens zum Symbol in der christlichen Kunst. ${ }^{3}$

Die mystische Sichtweise des Barockzeitalters konzentriert sich anstelle der sichtbaren, praktischen und daher für seine Denker unbedeutenden Erscheinungen der Welt auf die unsichtbaren, auf das ewig Wahre und dies, indem es die Welt in Symbolen darstellt und erklärt. Gleichzeitig ist der barocke Mensch aber in ständiger geistiger Verbindung mit den frühen Mystikern des Christentums und der biblischen Auslegung, indem deren Werke immer wieder gelesen werden, und ist daher empfindsam für das Wasser einerseits als eines der vier Grundelemente, andererseits auf die Bedeutung des Urelementes und der sich daraus ergebenden in erster Linie christlichen, in zweiter Linie griechisch-römischen - Symbolik.

\title{
Das Wasser und die Technik
}

Die Entwicklung der Physik als Wissenschaft, die moderne naturwissenschaftliche Denkweise beginnt sich generell mit dem Wasser als Mittel zu beschäftigen, als mit einem beherrschbaren, berechenbaren, durch Gesetze festmachbaren physikalischen Element. Bevor wir Beispiele für die Visibilisierung und Symbolik 
des Wassers in der Kunst des 18. Jhs suchen, muss zuerst die Frage geklärt werden, was der Mensch jenes Jahrhunderts über das Wasser als physikalisches Element und dessen Gesetze überhaupt wusste.

Als eine der bedeutenden Quellen ist dabei das handschriftliche, enzyklopädisch angelegte Lehrbuch eines Piaristen zu erachten, der darin die zeitgenössischen Ergebnisse der Naturwissenschaften zusammenzufassen versucht hatte: Lukács Mösch's Tätigkeit und sein handschriftliches Lehrbuch wartet noch darauf, von der Forschung entdeckt und aufgearbeitet zu werden. Mösch lebte und wirkte als lehrender Piarist zunächst in dem zur polnischen Provinz gehörigen Privigye, später in Podolin. Mösch spielte eine bedeutende Rolle in der Unabhängig-Werdung der ungarischen Provinz. Sein naturwissenschaftliches Interesse ist ungemein vielseitig und profund, seine militärwissenschaftlichen Kenntnisse trugen mittelbar vielleicht sogar zur Befreiung von Buda bei. Seine Werke erschienen wie gesagt nicht im Druck, er verfasste sie nur für den eigenen Gebrauch bzw. für seine Studenten. In den erhaltenen Handschriften trifft die Bemühung um Synthese des Apáczai Csere János und der ungarischen Karthesianer auf einen tief religiösen Anspruch und zugleich auf den Willen, gerade dieses Wissen über die Natur zu vermitteln. Das Skriptum Bibliothecae Mathematicae... Classis I-III. Anno 1684 enthält folgende Themenbereiche: angewandte Geometrie, Mechanik, theoretische und praktische Erdkunde, Akkustik, theoretische und praktischer Militärbaukunst, Chromatik, Hydrotechnik, Hydrostatik, Hydrographie. ${ }^{4}$ Der VII. Band von Möschs Lehrbuch trägt den Titel De Hydrotechnica et Hydrostatica denunque de Hydrographia, sive de Arte Aquaria, machinis atque Libratione Liquidorum. Er besteht aus drei Teilen und insgesamt 18 Kapiteln. Der erste Teil, De Hydrotechnica seu machinis Hydraulicis, enthält 11 der erwähnten 18 Kapitel. Hier wird nach Beschreibung von einigen Versuchen die Funktion einfacher Maschinen erklärt, die Art und Weise, wie das Wasser sich erhebt, Maschinen, die mit Auftriebskraft arbeiten, die Spritzdüsemaschinen und Maschinen, die aufgrund zusammengesetzter Kräfte arbeiten. Unter den diversen Maschinen des 9. Kapitels trägt eine den Namen Machina 8: Aquilea cesarea horodictica und sie zeigt vor, wie der Umriss einer Figur mit Hilfe einer Maschine beschrieben werden kann. Kapitel 11 beschäftigt sich mit dem Prinzip der Springbrunnen. So konnte offensichtlich sowohl das Konzept eines Gartens als auch das eines Bühnenbildes sehr wohl unter Zuhilfenahme von simplem technischem Mittelschulwissen verwirklicht werden. Dieses Wissen nutzte Mösch allerdings angesichts der Gefahr, die von den Türken ausging, vor allem für militärische Zwecke, wie auch sein Lebenslauf beweist. ${ }^{5}$

Die folgenden Illustrationen sind dem Werk Mösch's entnommen: 


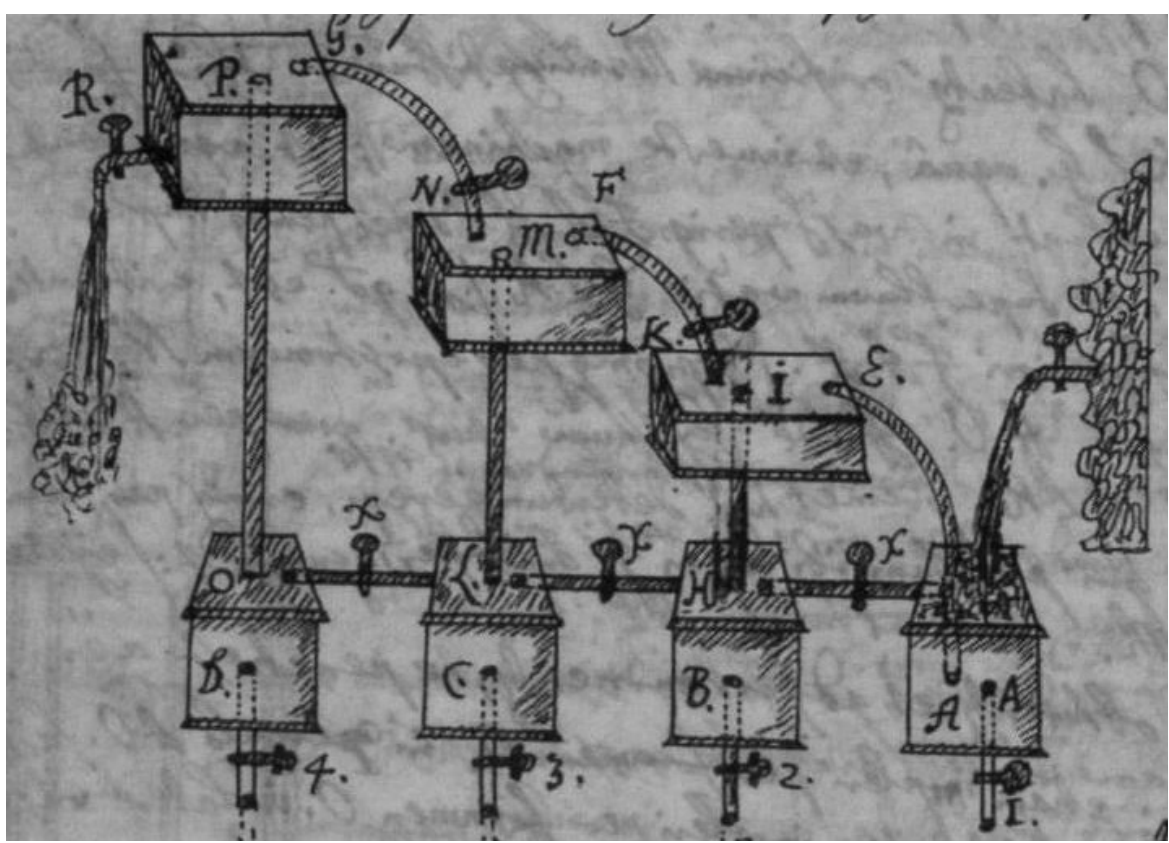

Bild 1. Hydraulische Einrichtung: Lucas Moesch Bibliothecae Mathematicae... Classis I-III. Anno 1684 (Budapest, Zentralbibliothek des Piaristenordens - Központi Piarista Levéltár)

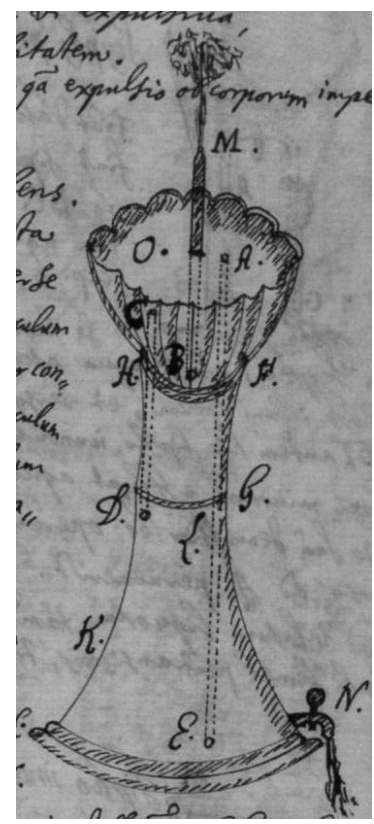

Bild 2. Technische Einrichtung: Lucas Moesch Bibliothecae Mathematicae... Classis I-III. Anno 1684 


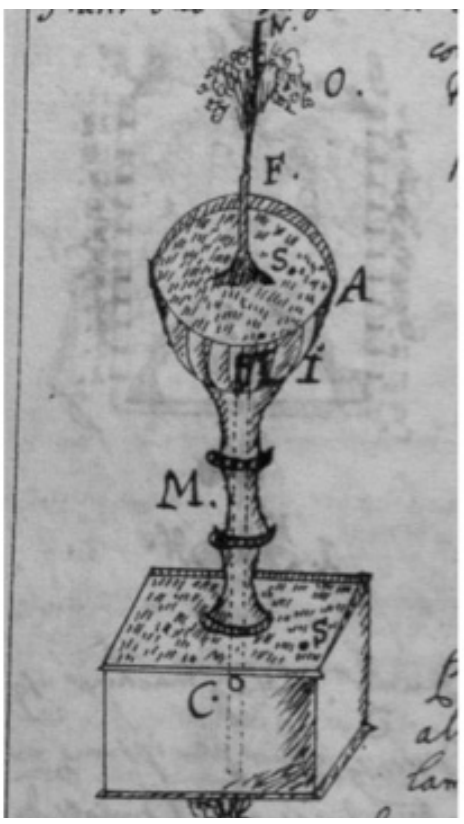

Bild 3. Das Prinzip des Springbrunnens: Lucas Moesch Bibliothecae Mathematicae... Classis I-III. Anno 1684

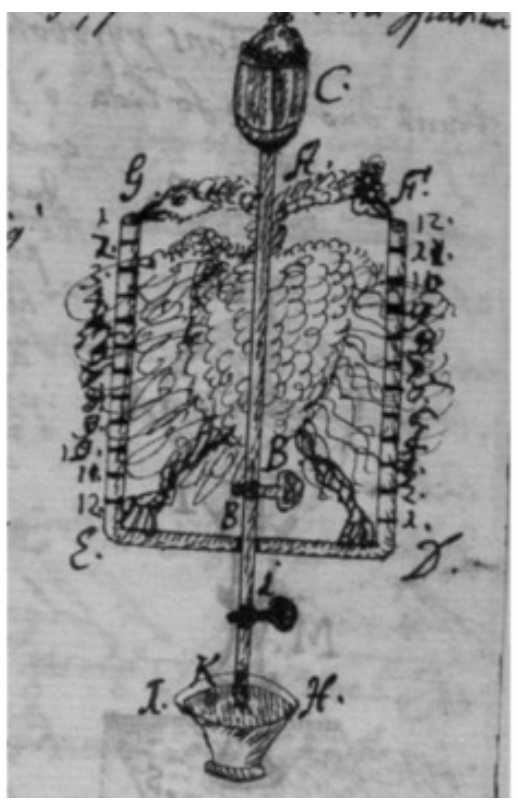

Bild 4. Hydraulische Einrichtung: Lucas Moesch Bibliothecae Mathematicae... Classis I-III. Anno 1684 
Durch diese Erkenntnisse wurde auch für das Königreich Ungarn der Grundstein für die dekorative Einbeziehung des Elements Wasser in die Gartenkunst aber auch in die Künste generell, zum Beispiel das Theater gelegt. Wasser konnte nicht nur zu Säuberung und anderen praktischen Zwecken dienen: es wurde gleichzeitig zur Attraktion.

\section{Das Wasser als Sehenswürdigkeit}

Wenn wir die Besonderheiten der Bühnenbilder des 18. Jhs beleuchten, so kommen wir nicht umhin, die Rolle des Wassers und dessen Symbolik näher zu untersuchen. Wir können dabei die Gartenarchitektur und deren Künste und auch deren Symbolik nicht außer Acht lassen, da sich das Theater des Barock nicht auf einen geschlossenen Raum als Bühne beschränkte, sondern auch häufig einen zweiten Aufführungsort, nämlich den Garten, den Park in den Mittelpunkt setzte. Ich möchte hier nicht über den Reichtum der barocken Gartenarchitektur berichten, sondern lediglich die Bedeutung des barocken Gartens als theatralischen Ortes hervorheben. Der Einfluss, den der Garten somit auf das geistig-kulturelle Leben gewann, ist evident. Und dies umso mehr, als im barocken Garten Wasser und dessen Einsatz als attraktives Element so wichtig war wie in keinem Zeitalter davor oder danach je wieder.

Der Garten ist von Beginn an die verkleinerte Version des Kosmos, die Verwirklichung des irdischen Paradieses, und dies nicht nur in der christlichen Mythologie, sondern auch in den Erklärungen der Welt der alten östlichen Kulturen. Sehr früh erscheint der Brunnen, die Quelle als zentrales Element des Gartens: die Quelle stand und steht als Symbol des Lebens. ${ }^{6}$ Dies kann natürlich auch aus dem alltäglichen Mangel an Wasser in diesen Regionen erklärt werden, aber der Kult, der sich um das Wasser herausbildete, weist darüber bei Weitem hinaus, und so dient Wasser oft als Zeichen für den Reichtum der Natur, als Ausdruck der Fruchtbarkeit.

Gleichzeitig ist das Wasser, die Quelle, das fließende Wasser aufgrund von biblischen Beschreibungen Träger diverser Symbole. Wenngleich in der offiziellen Theologie der Brunnen eindeutig das Symbol für Jesus ist, ist der Brunnen, das lebendige Wasser in der profanen Welt und in der Verwertung in der Kunst das Symbol für Maria. Diese Bedeutung geht auf das Hohelied Salomos zurück, ist also biblischen Ursprungs und - so sehr die Kirche sich auch dagegen verwehrte - weit verbreitet. So entwickelte sich im Zeitalter der Renaissance die neue Darstellung der rosenumwobenen Gärten, der so genannte hortus conclusus, in dessen Zentrum der ständig Wasser spendende, ständig in Bewegung seiende Springbrunnen steht. ${ }^{7}$ Der hortus conclusus ist auch in der barocken Gartenkultur 
zu finden, dort jedoch nicht mehr als Träger mittelalterlicher Symbolik sondern als Symbol für die varietas, das Vielbedeutende.

Ebenfalls in der Renaissance erscheint ein weiterer Garten-Typus, ein weltlicher Typus, der auch nicht ohne Wasser als tragendes Element auskommt, der hortus deliciarum, ein bis in die heutige Zeit geltender Topos. ${ }^{8}$

Diese beiden Gartentypen, der erste religiösen, der zweite weltlichen Ursprungs, weltlichen Freuden Ausdruck verleihend, sind im Zeitalter des Barock, in dem Mystik und Hedonismus Hand in Hand gehen, bereits untrennbar miteinander verbunden. Dies gilt auch für Ungarn, wenngleich wir über die Gepflogenheiten der Gartenkunst in Ungarn vor dem 19. Jh. noch zu wenig wissen.

Es ist aber bekannt, dass die in Gärten und Parks inszenierten Attraktionen auch im Kreise der ungarischen Aristokratie populär waren. Der Park von Eszterháza und die Beschreibungen, die uns darüber erhalten blieben, beweisen dies unmissverständlich. ${ }^{9}$ Es wurden aber bereits früher, im Jahr 1724 in Nordungarn durch die Jesuiten im Park des für seine Unterhaltungen so berühmten Palais Fourcontrasti so genannte theatrum navalis aufgeführt. Das Anwesen gehörte dem Erzbischofs von Erlau, Ferenc Barkóczy. Das Schloss wurde leider zerstört, man kennt es nur noch durch ein zeitgenössisches Gemälde religiösen Charakters, dessen Hintergrund das Gebäude darstellt. Auf dem Bild lässt sich jedoch gut erkennen, dass das Schloss inmitten einer großzügigen Anlage stand, und der Garten sehr wohl für oppulente barocke Theateraufführungen geeignet war.

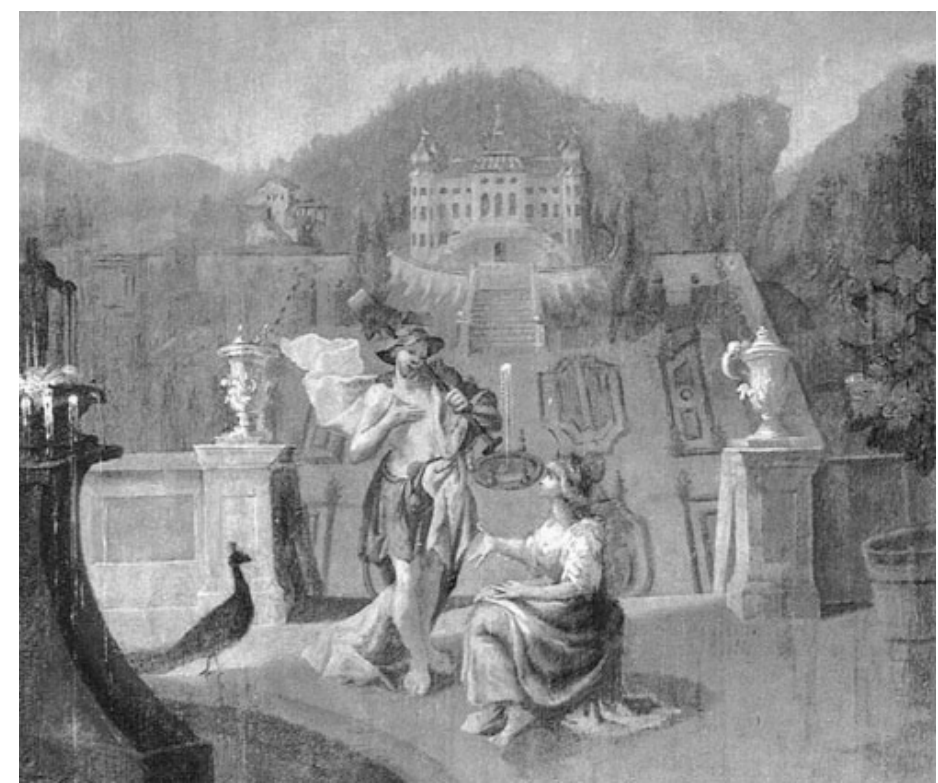

Bild 5. Das einmalige Schloss Fourcontrasti (Lucas Huetter: Noli me tangere 1753, Holz und Öl, Eger, Speisesaal der Barmherzigen Brüder) 
Auch auf den Besitztümern der Familie Esterházy in Cseklész trug ein wunderbares Schloss umgeben von einem Park zur Erfüllung der Repräsentationspflichten der Familie bei. Über den Teich, der sich inmitten des Gartens befand, weiß man folgendes:

... geschützt durch eine Allee gelangt man zum Tiergarten; es ist dies ein elyptisch angelegter Ort und der grün und rot bemalte Turm des kleinen Häuschens ist das Nest der fliegenden Tauben. Auf diesem Platz befinden sich noch vier weitere Häuschen. Zwischen den vier Häuschen befinden sich zu beiden Seiten je ein Springbrunnen, insgesamt daher zehn; sie erhalten ihr Wasser aus einem Turm im Wald, ein Mann benötigt einen halben Tag um den sechzigküblischen Behälter des Turmes anzufüllen, und diese Menge an Wasser versorgt der Springbrunnen sechs Stunden hindurch [...] Nun gelangt man zum Ententeich. Über eine schöne Brücke spaziert man auf einen elyptischen Platz mitten im Teich, der von einem großen Sonnenschirm geschützt ist, in dessen Schatten sich Sitzgelegenheiten anbieten. Im Wasser selbst befinden sich für die Enten rundum sieben grüne Häuschen. ${ }^{10}$

Das neben Pressburg gelegene Ivánka gehörte zu den Besitztümern des Grafen Antal Grassalkovich, der eben zu jener Zeit zum Herzog geworden war. Grassalkovich hatte die Kaiserin in seinem Anwesen in Gödöllő seinerzeit unter derart luxuriösen Umständen empfangen, dass die Legende um dieses Ereignis noch heute in der Tradition des Ortes lebendig ist. Damals hatten noch Feuerwerk und Lichtspiele eine Hauptattraktion dargestellt. Man weiß allerdings schon Genaueres über einen Empfang auf Ivánka, der vier Jahre später stattfand und auf dem das Wasser bereits eine große Rolle spielte. Es war gerade der Garten des Schlosses, der unter den Zeitgenossen besonders berühmt geworden ist. Da es sich um eine Sommerresidenz handelte, fiel diesem vor allem bei Großereignissen bedeutende Funktionen zu. Graf Antal Grassalkovich II. schien mit Herzog Miklós Esterházy I. und den Attraktionen, die im Garten zu Eszterháza geboten wurden, in Konkurrenz getreten zu sein. Als Erzherzogin Marie Christine und Prinz Albert von Sachsen-Teschen, das ungarische Statthalterpaar, am 20. September 1773 Ivánka besuchten, vermeldeten zeitgenössische Quellen folgendes:

Man begab sich in das holländische Lusthaus, und auf einen Glockenschlag erschienen auf dem Teich acht hübsch dekorierte Boote. Das erste trug die türkische Musik, das zweite war nach chinesischer Form gebaut, das dritte war blau angestrichen, das vierte trug den Sängerchor, das fünfte war nach venezianischer Art ganz schwarz gehalten und so weiter. Die Schiffleute waren dem Stil ihrer Schiffe entsprechend kostümiert. Unter Musikbegleitung umfuhren sie mehrere Male den Teich. ${ }^{11}$ 
Es ließen sich noch zahlreiche Beispiele anführen, die beweisen, welche Schlüsselfunktion dem Wasser in der Gestaltung des Gartens aber auch in der repräsentativen Theateraufführungskunst des Barock zugeeignet wurde. Das Wasser wurde im Zeitalter des Barock zum Träger symbolischer Inhalte - im Gegensatz zu seiner früheren (religiösen) Bedeutung und Funktion als eines der Grundelemente des Lebens. Voraussetzung dafür war jedoch die technische Entwicklung - hier vorgeführt durch das Handbuch von Mösch - und die damit einhergehenden Möglichkeiten, Wasser gestalterisch einzusetzen sowie die gesellschaftlichen Veränderungen generell, die eine Umdefinierung der symbolischen Werte, eine Profanisierung des Elements erlaubten.

\section{Anmerkungen}

Hamvas, Béla: Scientia Sacra. Az öskori emberiség szellemi hagyománya. Budapest: Magvetö, 1988, 454-455.

2 Huyghe, Edith und Huyghe, François-Bernard: Világképek. Az Univerzum ezeregy meséje Galilei elött. Budapest: Európa, 2000, 113.

3 Vanyó, László: Az ókeresztény müvészet szimbólumai. Budapest: Az Apostoli Szentszék Könyvkiadója, 1988, 162.

$4 \quad$ Zu seinem Lebenslauf siehe: Új Magyar Irodalomi Lexikon I-III. Hg. von Péter László. Budapest: Akadémiai Kiadó, 1994 sowie vor allem über seine heereswissenschaftlichen Aktivitäten Kilián, István: Házikáplán, tábori lelkész, tanár, hadmérnök. Emlékezés Moesch Lukácsra. Vigilia 1986/8, 582-588.

$5 \quad$ Kilián, ibid.

6 Siehe dazu: Szimbólumtár, jelképek, motívumok, témák az egyetemes és a magyar kultúrából. Hg. von Pál József und Újvári Edit. Budapest, Balassi, 2005, 263-264.

7 Kirchenbaum: Marienlexikon.

8 Szimbólumtár, 265.

9 Siehe dazu beispielsweise jüngst: Seidler, Andrea: Das ungarische Versailles - Eine Beschreibung des Schlosses Esterháza aus dem späten 18. Jahrhundert. In: Ágoston Zénó Bernád, Márta Csire, Andrea Seidler (Hg.), On the Road - Zwischen Kulturen Unterwegs. Wien: LIT Verlag 2009, 281-296.

10 Rapaics, Raymund: Magyar kertek. Királyi Magyar Egyetemi Nyomda, 1940, 112-113.

11 In: Staud Géza: Magyar kastélyszínházak. II. rész. Budapest: Színháztudományi Intézet, 1963, 27. und Staud: Adelstheater in Ungarn (18. und 19. Jahrhundert), Österreichische Akademie der Wissenschaften, Theater Geschichte Österreichs Band X: Donaumonarchie, Heft 2. Wien, 1977, 175. Forrás: Pressburger Zeitung 1773. szept. 22. 
